\title{
Teleophthalmology for Eye Care during Coronavirus Disease 2019 Pandemic: Opportunities and Challenges
}

\author{
Sareh Keshvardoost ${ }^{1}$, Kambiz Bahaadinbeigy ${ }^{2}$ and Farhad Fatehi ${ }^{3,4, *}$ \\ ${ }^{1}$ MSc in Medical Informatics, Medical Informatics Research Centre, Institute for Future Studies in Health, Kerman University of Medical Sciences, Kerman, Iran \\ 2 MD, PhD, Associate Professor, PhD in Medical Informatics, Health Services Management Research Centre, Institute for Future Studies in Health, Kerman \\ University of Medical Sciences, Kerman, Iran \\ ${ }^{3}$ MD, PhD, Research Fellow, School of Psychological Sciences, Monash University, Melbourne, Australia \\ ${ }^{4}$ Honorary Fellow, Centre for Online Health, The University of Queensland, Brisbane, Australia
}

* Corresponding author: Farhad Fatehi, Research Fellow, School of Psychological Sciences, Monash University, Melbourne, Australi; Honorary Fellow, Centre for Online Health, The University of Queensland, Brisbane, Australia. Tel: 0061430992014; Email: f.fatehi@uq.edu.au,ffatehi@gmail.com

Received 2020 October 20; Revised 2020 November 09; Accepted 2020 November 15

\begin{abstract}
The coronavirus disease 2019 (COVID-19) pandemic illustrated the significance of using telemedicine for medical services to minimize the risk of infection more than ever. The implementation of telemedicine in the realm of eye expertise (teleophthalmology) has made it possible for both ophthalmic patients and COVID-19 positive cases to be taken care of in different ways of prevention, diagnosis, treatment, and follow-up. This study aimed to discuss the ophthalmology challenges during the COVID-19 outbreak based on existing evidence, followed by proposing some solutions for utilizing telemedicine.

Keywords: Coronavirus, COVID-19, Telemedicine, Teleophthalmology, Virtual ophthalmology
\end{abstract}

\section{Background}

Coronavirus Disease 2019 (COVID-19) pandemic became a global health crisis in early 2020. It appeared in December 2019 in Wuhan, China, and spread out rapidly in most parts of the world. As of August 4, 2020, the total number of confirmed cases of this disease exceeded 18 million and accounted for more than 698,000 deaths worldwide (1). Although fever, dry cough, and respiratory problems are the most common symptoms of COVID-19, there is also evidence of ocular manifestations of the COVID-19 (2). In order to reduce the spread of the virus, the American Academy of Ophthalmology has recommended that examination services should be provided to emergency cases only, and nonemergency ophthalmology services should be stopped (2). In response to calls for implementing measures to reduce the risk of infection for patients and healthcare providers, the use of telemedicine rapidly expanded during the COVID-19 pandemic.

The feasibility and efficacy of telemedicine in ophthalmology (Teleophthalmology) have been established in several studies (3). Teleophthalmology has been successfully utilized in both inpatient and outpatient settings, as well as various socioeconomic settings. While most of the successful initiatives have focused so far on the screening and early detection of ophthalmic diseases, Teleophthalmology has shown potentials for treatment and follow-up of the patients as well. Obviously, the main advantage of Teleophthalmology is the elimination of the patients' referral to hospitals or healthcare centers (4).

During the COVID-19 crisis, the health systems of many countries experienced an unprecedented burden of managing patients along with the provision of the highest level of protection for their personnel. The proximity of patients, who may be a confirmed or suspected case of COVID-19, and health professionals during most of the eye examinations put the staff at a high risk of infection. Since one of the main routes of COVID-19 infection is through the eyes, additional safety measures are required for eye care specialists when managing patients. Considering this, wearing face shields in addition to other personal protection devices, such as masks and gloves, deemed necessary, yet the risk of infection for eye specialists remained higher than most of the other specialties. Therefore, it is of high importance to find a solution for keeping patients and clinicians away when eye examinations are required. Similarly, the risk of crosscontaminations for the people who visit hospitals and healthcare centers has been reported to be high.

This issue has an impact on lots of people who require a medical service during the COVID-19 crisis, especially patients with chronic diseases as they might prefer to postpone their appointments. It has been stated that the fear of being infected with COVID19 has killed more people than the disease itself. There are reports that many patients with eye disease have developed complications or worsened their condition because of their reluctance to show up for their appointments or seek medical advice when it was needed. Research shows that the ocular 
manifestations of COVID-19 are not very frequent (5). The most common ocular symptoms of these patients are sore eyes, itching, foreign body sensation, tearing, redness, dry eyes, eye secretions, and floaters (6). In some cases, ocular manifestations developed before respiratory symptoms of COVID-19 (7).

There is a high probability transmission risk of coronavirus diseases in ophthalmology and optometry clinics due to the routine use of ophthalmic equipment (i.e., slit lamp devices and ophthalmoscopes), proximity of the physician's face to the patient during the examination, high number of attended patients, and patient's referral to different departments for receiving counseling. Since the nasopharynx and upper respiratory tract are the main sites of viral infections, conjunctivitis may also be the first manifestation of coronavirus disease. Although many cases of conjunctivitis are not necessarily viral, ophthalmologists come inevitably in close contact with infected people and are at risk of infection.

Li Wenliang, a Chinese ophthalmologist, was the first physician who warned about a new respiratory virus similar to the severe acute respiratory syndrome. According to his report, he was infected with COVID-19 after examining a patient with glaucoma symptoms. He eventually died in February 2020 (8).

Referrals to ophthalmology clinics increase the risk of viral infection for patients, especially the elderly, diabetics, glaucoma patients, and people with hypertension, who are among the most frequent visitors by ophthalmologists. Studies have shown that about $25 \%$ of the people who are hospitalized with severe COVID-19 infection have diabetes. Since hyperglycemia weakens the immune system, the effects of the disease on these patients are greater and more serious, compared to nondiabetic patients (9). Therefore, in an epidemic situation, it is important to isolate the infected persons to prevent the virus from spreading to others. Teleophthalmology can be of help to minimize the contact of infected patients with healthy people and healthcare staff by reducing unnecessary referrals.

Although many patients are required to be referred to other clinics, they avoid it due to the fear of being infected with the virus. Moreover, since restrictions are imposed between cities, the patients can not travel to other cities to receive special care, and physicians are not willing to provide patients (i.e., glaucoma patients and those with sudden vision loss whose eyesight deteriorates if left untreated) with services in this situation. Moreover, regarding the retinopathy of prematurity in neonates, the diagnosis and treatment have a golden time (the first three to four weeks of birth), and newborns with retinopathy need regular weekly or monthly checkups; otherwise, it leads to blindness.
Due to the current situation, parents may not bring their infants to the medical centers. Sunny (MD, Department of Ophthalmology, Tung Wah Eastern Hospital, Hong Kong, China) reported the measures taken by the public emergency in a hospital-based general ophthalmology clinic during the COVID-19 outbreak. Phone calls were made to 547 patients, and they were asked about their absence to continue the treatment process. The results of this study showed an increased number of not attended patients, as more than half of the patients $(44.6 \%)$ did not refer to the hospital due to COVID-19 and fear of infection. Moreover, about $26 \%$ of them $(n=142)$ were glaucoma patients who required regular medication, and $19.6 \% \quad(n=107)$ of the patients required monitoring and timely injection of anti-vascular endothelial growth factor due to macular degeneration (10).

In these cases, the patients could communicate with their physicians through the Teleophthalmology program. The advantages of this approach include reducing the risk of COVID-19 infection by preventing the presence of the person, checking the patient's eye condition, and taking the necessary measures. In fact, the decision-making process is performed using virtual triage in order to prioritize the patients to receive ophthalmology services. Following that, regarding the patient's condition (emergency or not), services are provided in person or virtually.

A study was conducted at the University of Pittsburgh, Pittsburgh, Pennsylvania, on the experiences in the ophthalmology department about COVID-19 and its impact on the practice (11). In the adopted approach, the patient triage was performed virtually. From March 19 to April 30, the patients were classified into three groups of low (next three to six months' appointment schedule), moderate (manageable with online visits), and high risk (urgent need for in-person visits). They reported that the virtual triage could be helpful to better classify the patients for any clinical decision-making process. However, a proportion of patients required face-to-face urgent visits, and depending on the type of the eye problem, $1.9 \%$ (glaucoma)-17.6\% (retina) of the patients could only be managed by Teleophthalmology and online visits.

Moreover, based on the results of the aforementioned study, decision-making in ophthalmology depends on the use of medical equipment, such as slit-lamps and fundus camera. Online visits can be used to triage patients, follow them up, prescribe medication, monitor their conditions, and answer their questions.

During this public health crisis in which most of the countries are challenged with COVID-19 outbreak across the world, Teleophthalmology is an appropriate and effective tool for patient triage and delivery of eye care. This program can assess the ocular symptoms caused by COVID-19, such as 
sub-conjunctival hemorrhages and conjunctivitis. Prevention of the patients' referral will reduce the transmission of disease, and Teleophthalmology can manage people who do not need to be attended in clinics in-person and defer their appointments to a suitable time. Furthermore, it can prevent the worsening of the eye problems by enabling the timely diagnosis of high-risk patients who will not otherwise visit their ophthalmologists.

\section{Acknowledgments}

The authors express their gratitude to Dr. Hashem Shadman (Retina Subspecialty) for his valuable comments.

\section{Footnotes}

Authors' Contribution: All authors have had a significant contribution in drafting, writing up, and editing the manuscript.

Conflict of Interests: The authors have no conflict of interest to declare.

Ethical Approval: Not applicable.

Funding/Support: Nil.

Informed consent: Not applicable.

\section{References}

1. COVID-19 Coronavirus Pandemic. Worldometers. Available at:URL: https://www.worldometers.info/coronavirus/;2020.

2. Recommendations for urgent and nonurgent patient care.
American Academy of Ophthalmology. Available at:URL: https://www.aao.org/headline/new-recommendationsurgent-nonurgent-patient-care.

3. Labiris G, Panagiotopoulou EK, Kozobolis VP. A systematic review of teleophthalmological studies in Europe. Int $J$ Ophthalmol. 2018;11(2):314-25. doi: 10.18240/ijo.2018.02.22. [PubMed: 29487825].

4. Hanson C, Tennant MT, Rudnisky CJ. Optometric referrals to retina specialists: evaluation and triage via teleophthalmology. Telemed J E Health. 2008;14(5):441-5. doi: 10.1089/tmj.2007. 0068. [PubMed: 18578678].

5. Guan WJ, Ni ZY, Hu Y, Liang WH, Ou CQ, He JX, et al. Clinical characteristics of coronavirus disease 2019 in China. $N$ Engl J Med. 2020;382(18):1708-20. doi: 10.1056/NEJMoa2002032. [PubMed: 32109013].

6. Hong N, Yu W, Xia J, Shen Y, Yap M, Han W, et al. Evaluation of ocular symptoms and tropism of SARS-CoV-2 in patients confirmed with COVID-19. Acta Ophthalmol. 2020;10.1111/ aos.14445. doi: 10.1111/aos.14445. [PubMed: 32336042].

7. Daruich A, Martin D, Bremond-Gignac D. Ocular manifestation as first sign of Coronavirus Disease 2019 (COVID-19): interest of telemedicine during the pandemic context. J Fr Ophtalmol. 2020;43(5):389-91. doi: 10.1016/j.jfo.2020.04.002. [PubMed: 32334847].

8. Chinese Doctor, Silenced After Warning of Outbreak, Dies From Coronavirus. The New York Times. Available at:URL: https://www.nytimes.com/2020/02/06/world/asia/chinesedoctor-Li-Wenliang-coronavirus.html; 2020.

9. COVID-19 and Diabetes. WebMD. Available at:URL: https://www.webmd.com/diabetes/diabetes-and-coronavirus; 2020.

10. Au S. A surge in eye clinic nonattendance under 2019 novel coronavirus outbreak. Indian J Ophthalmol. 2020;68(5):948. doi: 10.4103/ijo.IJO_673_20. [PubMed: 32317502].

11. Williams AM, Kalra G, Commiskey PW, Bowers EM, Rudolph BR, Pitcher MD. Ophthalmology practice during the coronavirus disease 2019 pandemic: The University of Pittsburgh Experience in promoting clinic safety and embracing video visits. Ophthalmol Ther. 2020;9(3):1-9. doi: 10.1007/s40123-020-00255-9. [PubMed: 32377502]. 